\title{
Review of: "Tranilast for Advanced Heart Failure in Patients with Muscular Dystrophy: A Single-Arm, Open-Label, Multicenter Study"
}

Ugur Canpolat

Potential competing interests: The author(s) declared that no potential competing interests exist.

Manuscript title. Tranilast for Advanced Heart Failure in Patients with Muscular Dystrophy: A Single-Arm, Open-Label, Multicenter Study

In the current study, the authors assessed the safety and efficacy of tranilast on heart failure outcomes in MD patients. The paper was well written in general. The study sample size was small based on the selection of proper subjects accordingly. Please include the LVEF\% of the patients. The follow-up interval was short to assess the efficacy in regard to heart failure outcomes, particularly for adverse events like mortality. 